\title{
Capacity Model Research of Aluminum Conductor Composite Core(ACCC) Conductor
}

\author{
Li Qin ${ }^{1}$, Guangyu Zhou ${ }^{1}$, Bing Tang ${ }^{1}$ a* \\ ${ }^{1}$ Northeast Dianli University Shcool Of Civil Engineering and Architecture, Jilin, China \\ a537728541@qq.com
}

Keywords: Aluminum Conductor Composite Core conductor current-capacity increasing temperature finite element modelling

\begin{abstract}
As the rapid development of economy and electricity, it is particularly important to research about improving the exiting transmission capacity of transmission lion on the premise of safety. Compared with conventional conductor, ACCC conductor has low sag, high current carrying capacity, but the design of the carbon fiber wire still routinely traverse design method, it does not give full reflect to the characteristics of the carbon fiber wire. According to the theory equilibrium principle, Ansys the main influencing factors of carrying capacity. Research the relationship of temperature and carrying capacity by using the finite element simulation and regression analysis. It has practical value as this model effectively reduce the complexity and reduced the measuring equipment.
\end{abstract}

\section{Introduction}

Carbon fiber wire (ACCC) inside is made of carbon fiber and glass fiber coated composite core, external tightly arranged by trapezoidal section soft aluminum wire. Compared with the conventional wire Accc has high strength, load flow, thermal expansion coefficient, high conductivity, loss of small, light weight and corrosion resistance etc. advantages, get gradually popularize in the power system [1-3].

Transmission line in the actual design, so as to prevent the load is too large to produce thermal heat capacity level set by the fault lines is a kind of static and conservative values .This limit is based on the most severe weather conditions for the maintenance of the line to the ground safe distance, but such bad weather conditions rarely occur. This has resulted in the large number of cases, the carrying capacity of the actual value of the design value of the load flow. Appropriate allowed beyond the security and stability of some does not affect the line, at the same time, give full play to the conductor current-carrying performance [4].

Monitoring capacity of the capacity of the maximum allowable load flow of the conductor is calculated by the use of the mathematical model, which is based on the actual data. For dynamic research domestic and overseas mostly in the dynamic monitoring system of the overall research, equipment, optimization of monitoring system to monitor environmental factors such as temperature, wind speed, sunshine, tension, because of monitoring equipment cost is high, the construction inconvenience limit and didn't get the promotion Therefore, it is very important to study the line capacity model with less monitoring and high accuracy [5-8].

\section{Getting Started}

The Steady-state Heat Equation. The conductor and the external environment always have heat exchange, when the wire temperature is basically stable, the system reaches the steady state. The heat balance equation is as follows:

$$
\mathrm{E}_{\mathrm{g}}+\mathrm{E}_{\mathrm{st}}=\mathrm{E}_{\text {conv }}+\mathrm{E}_{\mathrm{rad}}
$$


where $E_{g}=$ the energy of the length of the lead unit, and the $E_{s t}=$ power of the length of the wire unit $\mathrm{W} / \mathrm{m} ; \mathrm{E}_{\mathrm{cov}}=$ conductor unit length of the convection cooling power; $\mathrm{E}_{\mathrm{rad}}=\mathrm{r}$ the conductor unit length of the radiation heat power.

$$
\begin{aligned}
& E_{g}=I^{2} R \\
& \mathrm{E}_{\mathrm{rad}}=\pi \delta D\left[\left(\theta+t_{a}+273\right)^{4}-\left(t_{a}+273\right)^{4}\right] \\
& \mathrm{E}_{\mathrm{conv}}=0.57 \pi \lambda \theta \mathrm{R}_{\mathrm{s}}^{0.485}=9.92 \theta(\mathrm{VD})^{0.485} \\
& \mathrm{E}_{\mathrm{st}}=\alpha_{\mathrm{s}} \mathrm{I}_{\mathrm{s}} \mathrm{A}
\end{aligned}
$$

where, $\lambda=$ Heat transfer coefficient of air layer for air surface; Re=Reynolds number; $\mathrm{R}=\mathrm{AC}$ resistance of unit length wire; $\theta=$ temperature rise for the lead surface; $\varepsilon=$ the radiation coefficient (bright new 0.23 0.43, old black line 0.9 0.95): $\mathrm{I}=$ wire current; $\mathrm{t}_{\mathrm{a}}=$ is the ambient temperature. $\mathrm{S}=$ Stephen - Bo leboltzmann constant, $\mathrm{S}=5.67 \times 10^{-8}$;

The calculation conditions of transmission line in the world are as follows: the ambient temperature is 40 , the wind speed is $0.5 \mathrm{~m} / \mathrm{s}$, and the sunshine intensity is $1000 \mathrm{w} / \mathrm{m}^{2}$, and the absorption coefficient is 0.9 , and the absorption coefficient is 0.9 The above parameters into the formulas (1):

$$
I^{2}=\frac{9.92 \theta(V D)^{0495}+\pi \varepsilon S D\left[\left(\theta+t_{a}+273\right)^{4}-\left(t_{a}+273\right)^{4}\right]-\alpha_{x} I_{x} A}{R}
$$

The Influencing Factors of Carrying Capacity. In order to determine the influence of the parameters in the formula (2) on the traffic flow, the matlab program is compiled. Calculation conditions: the change of a single variable, the other variables are used in the national standard of the calculation conditions, Fig. 1-4 derived formula in the impact of the various factors on the load flow.

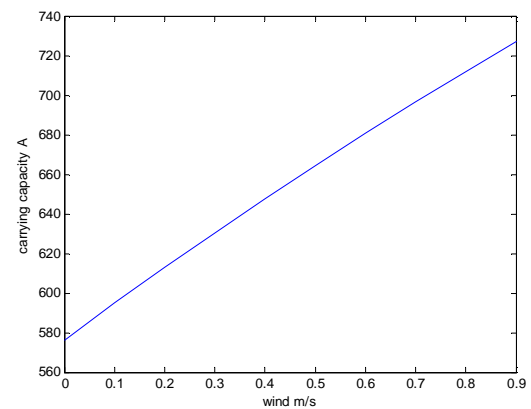

Fig. 1 Wind.

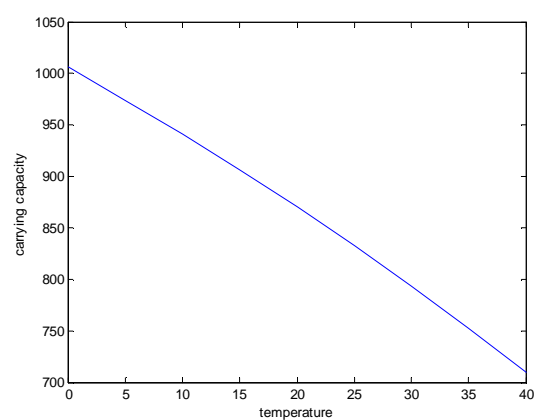

Fig. 3 Environment.

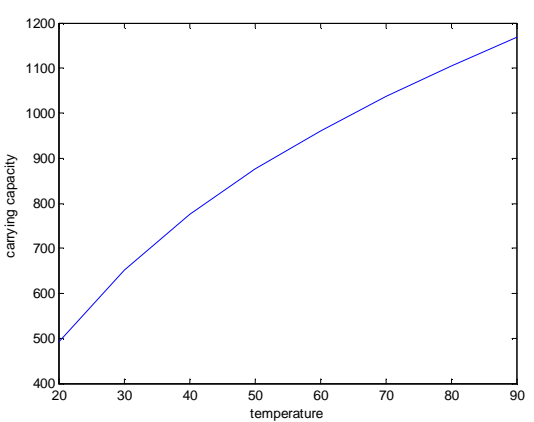

Fig. 2 Temperature.

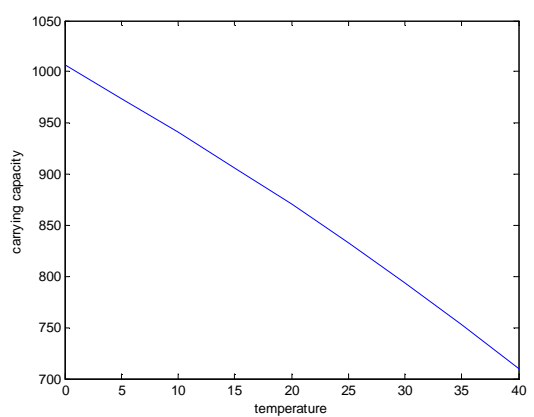

Fig. 4 Radiation coefficient.

Fig. 1-4 shows that the wind speed from $0 \mathrm{~m} / \mathrm{s}$ to $5 \mathrm{~m} / \mathrm{s}$, the load flow of the lead increased by $500 \mathrm{~A}$, and the wind speed calculated by $0.5 \mathrm{~m} / \mathrm{s}$ is not accurate $300 \mathrm{~A}$. With the increase of the ambient temperature, the load flow rate decreased rapidly, the environmental temperature increased from 0 to 40, the load flow rate decreased by nearly 300A. According to the provisions of the 40 degrees Celsius in the calculation of the load flow is less than the actual leadLead temperature 
increased, the load flow increased significantly, increasing the $800 \mathrm{~A}$ or so, the conductor temperature had a significant effect on the carrying capacity; Sunshine intensity changed from 0 to 1000 , the load flow was reduced by about 90A, and the sunshine intensity had little effect on the carrying capacity; the heat absorption coefficient and the radiation coefficient change of the lead wire, the load flow rate is about $60 \mathrm{~A}$, which has little influence on the carrying capacity of the conductor.

To sum up, the factors that affect the carrying capacity of the wire are $t$ wind speed, environment temperature, conductor temperature and the wire resistance. And wind speed, environment temperature is determined by the environmental conditions, when the wire mesh is fixed, wire temperature and resistance are determined by the wire itself, the resistance is related to the temperature change:

$$
I^{2}=\frac{a_{1} T^{4}+a_{2} T^{3}+a_{3} T^{2}+a_{4} T+a_{5}}{a_{6} T+a_{7}}
$$

\section{Numerical Simulation and Regression Analysis}

Ansys Numerical Simulation. Because the wire is symmetrically distributed. In Ansys to establish to outer conductor surface position of the center of a circle as the origin of coordinates of one of four wire 3D model, internal conductor three-dimensional heat transfer equations, initial boundary conditions, conditions.

$$
\left\{\begin{array}{l}
\lambda\left(\frac{\partial^{2} t}{\partial x^{2}}+\frac{\partial^{2} t}{\partial y^{2}}+\frac{\partial^{2} t}{\partial z^{2}}\right)+\varphi_{1}+\varphi_{2}=\rho c \frac{\partial t}{\partial \tau} \\
\left.t\right|_{\tau_{0}}=t_{0} \\
q=-\left.\lambda \frac{\partial t}{\partial n}\right|_{s}=a_{c o n}\left(t_{w}-t_{f}\right)+q_{r}
\end{array}\right.
$$

where, $t=$ wire temperature; $x, y, z$ for space rectangular coordinates: $\varphi=$ Material per unit volume heating rate; $\rho=$ Density of matter; $c=$ Specific heat capacity; $\tau=$ time; $t_{0}$ Initial temperature; $q=$ Heat flux density of a point on the outer surface; $a_{c o n}=$ Convection heat transfer coefficient; $t_{f}=$ Ambient temperature; $q r=$ Radiant heat flux density.

\section{Heating Rate Calculation.}

$$
\left\{\begin{array}{l}
\varphi_{1}=I_{1}^{2} R_{1} / v_{1} \\
\varphi_{2}=\left(I_{2}^{2} R_{2}+\alpha_{s} I_{s} D\right) / v_{2}
\end{array}\right.
$$

where, $\varphi_{1}, \varphi_{2}$, through the heating power after power per unit length, core wire; $v_{1}, v_{2}=$ the core and aluminum volume; $I=$ Electric current; $R=$ unit length resistance; $D=$ calculated outside diameter of conductor; $I_{s}=$ the sunshine intensity.

Simulation Results. According to the ambient temperature, the heat absorption coefficient of conductor is 0.9 , the radiation coefficient is 0.9 , the wind speed is $0 \mathrm{~m} / \mathrm{s}$, simulate ACCC $300 / 50$ carbon fiber conductor and LGJ30/50 is under natural convection.

The follow chart shows that the temperature of the conductor increases with the increase of the load flow, and it is lower than the natural convection in the same load flow. This is in line with the actual situation. By the same load flow, the carbon fiber lead temperature is lower, which is due to the external carbon fiber wire for soft aluminum, the higher conductivity, low resistance, through the same load flow when the heat generated by small. 
Table 1. Relationship between temperature and load flow of steel core aluminum conductor under natural convection.

\begin{tabular}{cccc}
\hline $\mathrm{I}(\mathrm{A})$ & $\varphi 1 \mathrm{w} / \mathrm{m} 3$ & $\varphi 2 \mathrm{w} / \mathrm{m} 3$ & $\mathrm{t}\left({ }^{\circ} \mathrm{C}\right)$ \\
\hline 110 & 484.5779174 & 56120.59 & 43.88 \\
160 & 1025.222701 & 59812.17 & 45.21 \\
210 & 1766.106294 & 64871.01 & 46.99 \\
260 & 2707.228695 & 71297.09 & 49.2 \\
310 & 3848.589906 & 79090.44 & 51.85 \\
360 & 5190.189925 & 88251.03 & 54.9 \\
410 & 6732.028753 & 98778.87 & 58.3 \\
460 & 8474.10639 & 110673.97 & 62 \\
510 & 10416.42284 & 123936.32 & 66.1 \\
560 & 12558.97809 & 138565.92 & 70.46 \\
610 & 14901.77215 & 154562.78 & 75.1 \\
660 & 17444.80503 & 171926.88 & 79.9 \\
\hline
\end{tabular}

Table 2. Relationship between temperature and load flow of Aluminum conductor composite core conductor under natural convection.

\begin{tabular}{ccc}
\hline $\mathrm{I}(\mathrm{A})$ & $\varphi \mathrm{w} / \mathrm{m} 3$ & $\mathrm{t}\left({ }^{\circ} \mathrm{C}\right)$ \\
\hline 150 & 57536.28 & 43.57 \\
200 & 61363.22 & 44.89 \\
250 & 66283.58 & 46.54 \\
300 & 72297.35 & 48.51 \\
350 & 79404.5 & 50.79 \\
900 & 229748.79 & 92.43 \\
950 & 249976.93 & 97.28 \\
1000 & 271298.48 & 102.98 \\
1050 & 293713.45 & 109.83 \\
1100 & 317221.82 & 117.02 \\
1150 & 341823.61 & 124.55 \\
\hline
\end{tabular}

\section{Regression Analysis}

For the type (3) of each coefficient, using the least square method to carry out matlab fitting, according to the simulation data to draw scatter plot, using the formula (3) for the original equation, drawing the fitting curve, the fitting result is as follows:

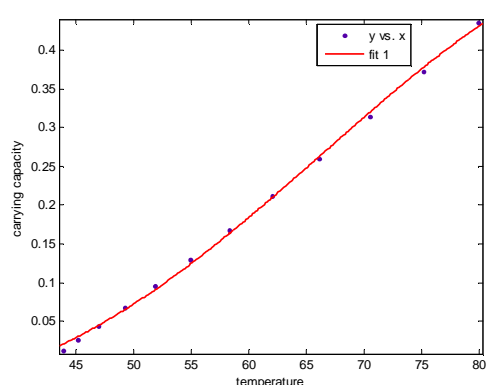

Fig. 5 Temperature of LGJ

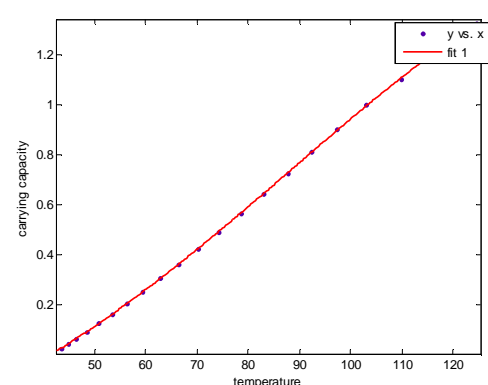

Fig. 6 Temperature of Accc. 
Fig. 5 the scattered point and curve fitting of the temperature of the conductor under the natural convection of the steel core aluminum strand, Fig. 6 the dispersion point and curve fitting of the temperature load flow in the natural convection of carbon fiber conductor. From the fitting situation, no matter which kind of traverse formula (3) can be a good reflection of the relationship between temperature and load flow, the maximum allowable transmission limit, in the case of electric power, can adjust the load flow, so as to achieve the purpose of increasing capacity.

\section{Conclusions}

The new carbon fiber wire than traditional steel core aluminum stranded wire has a higher operating temperature, small resistance, carrying capacity is bigger, according to the existing design under the worst conditions, and can not give full play to the good performance of carbon fiber wire. Through the finite element analysis and regression analysis, the paper shows that the load flow temperature model can reflect the relationship between the carbon fiber lead flow and wire temperature. Through the actual data, we can calculate the limit transmission capacity of carbon fiber wires, so as to achieve the purpose of the wire capacity, reduce the number of necessary measurement device, the measurement is less, the cost is low, and it will be widely used.

\section{References}

[1] X. M. Liang, J. Yu, C. Y. You. A survey of aluminium conductor with reinforced core of composite material, Power Syst. Technol. 30(19) (2006) 1-6.

[2] X. Hu, Application of ACCC Wire on Overhead Power Transmission Line. An Hui Electr. Power, 27(01) (2006) 51-53.

[3] Z. W. He, X. Chen, et al. Research and application overview of ACCC conductor in China. Electr. Power Constr. 31(4) (2010) 90-93.

[4] N. Yang, Prospect analysis of ACCC aluminum conductor technology in China. Electrotech. Appl. 27(5) (2008) 50-52.

[5] W. D. Jiang, Y. Zhang, Application of ACCC conductor in line transformation project. East China Electr. Power, 37(3) (2009) 418-421.

[6] Z. W. You, A. J. Zhu, Y. X. Pan, et al. Application analysis of ACCC conductor in Shanghai power grid. East China Electr. Power. 37(8) (2009) 1292-1295.

[7] D. Liang, S. P. Deng et al. Situation of technology research and engineering application for aluminum conductor composite core reinforced cable. New Chem. Mater. 39(SI) (2011) 13-17.

[8] D. A. Douglass, A. A. Edris, Field studies of dynamic thermal rating methods for overhead lines. In Proceedings of the IEEE Power Engineering Society Transmission and Distribution Conference, NewOrleans, USA, 2 (1999) 842-851. 